\section{Commentary: Low tidal volume ventilation: How low can you go?}

\author{
Justin Karush, DO, and Michael Liptay, MD
}

Acute lung injury is a common cause of morbidity and mortality after thoracic surgery, and the pathophysiology of this is complex and multifactorial. Regarding one-lung ventilation (OLV), causative mechanisms believed to trigger injury include large, nonphysiologic tidal volumes to the ventilated lung, as well as oxidative stress due to hyperperfusion. ${ }^{1}$ The nonventilated lung is also at risk to injury from surgical manipulation, as well as re-expansion ischemia-reperfusion injury promoting inflammatory cytokine release. ${ }^{2}$

Protective ventilation strategies are well recognized and widely used in critical care. They include lower tidal volumes and greater positive end-expiratory pressure to minimize barotrauma and improve recruitment of noncompliant lungs. ${ }^{3}$ This is frequently at the expense of optimal ventilation, with resultant hypercapnia. In the setting of OLV, a similar strategy is frequently employed, with lower tidal volumes $(4-6 \mathrm{~mL} / \mathrm{kg}$ ) and physiologic positive endexpiratory pressure $\left(5-10 \mathrm{cmH}_{2} \mathrm{O}\right)$. There is no consensus whether this strategy provides equivalent oxygenation or improves outcomes, and no single-ventilation strategy during OLV has been unanimously favored over another.

In this issue of the Journal, Peel and colleagues ${ }^{4}$ present a systematic review and meta-analysis to assess the effect of "conventional" tidal volume versus "low" tidal volume on oxygenation, compliance, and postoperative complications, including both lung resection and esophagectomy. Their review identified 18 studies and included 3693 total patients. While there was no standardized definition of low or conventional tidal volume, the mean volumes analyzed were 5.6 versus $8.1 \mathrm{~mL} / \mathrm{kg}$, respectively. The authors identified no significant differences in arterial oxygen tension, $\mathrm{P} / \mathrm{F}$ ratio (arterial oxygen tension/inspired oxygen fraction), or

\footnotetext{
From the Department of Thoracic Surgery, Rush University Medical Center, Chicago, Ill.

Disclosures: The authors reported no conflicts of interest.

The Journal policy requires editors and reviewers to disclose conflicts of interest and to decline handling or reviewing manuscripts for which they may have a conflict of interest. The editors and reviewers of this article have no conflicts of interest.

Received for publication Dec 23, 2020; revisions received Dec 23, 2020; accepted for publication Dec 24, 2020; available ahead of print Jan 10, 2021.

Address for reprints: Michael Liptay, MD, University Thoracic Surgeons, $1725 \mathrm{~W}$ Harrison St, Suite 774, Chicago, IL 60612 (E-mail: Michael_Liptay@rush.edu). J Thorac Cardiovasc Surg 2022;163:1586-7

$0022-5223 / \$ 36.00$

Copyright (C) 2021 by The American Association for Thoracic Surgery http://dx.doi.org/10.1016/j.jtcvs.2020.12.124
}

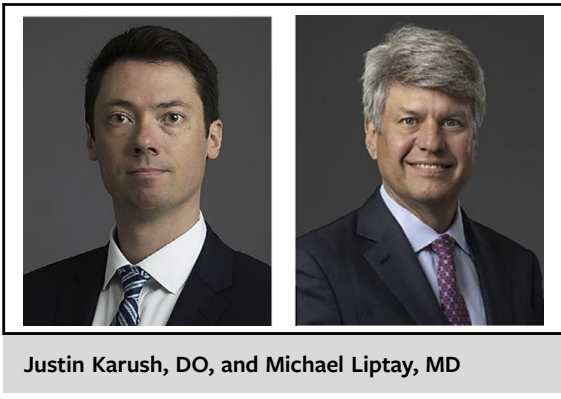

\author{
CENTRAL MESSAGE \\ Low tidal volume during one- \\ lung ventilation provides equiva- \\ lent oxygenation as conventional \\ tidal volume and results in lower \\ rates of pulmonary \\ complications.
}

compliance between the 2 groups. Outcome analysis demonstrated a significant reduction in pulmonary complications in the low tidal volume cohort.

The authors should be commended on their excellent analysis, strengthening the level of evidence for lungprotective strategies, which is currently considered moderate according to the European Society of Thoracic Surgeons enhanced recovery after lung surgery guidelines. ${ }^{5} \mathrm{We}$ consider these measures to be critical not only for the physiologic benefit they provide but also for the conduct of a video-assisted thoracoscopic surgery lung resection itself.

As mentioned previously, many factors contribute to minimizing complications after thoracic surgery. Notably, this includes smoking cessation, fluid management, regional anesthesia, early ambulation, pulmonary hygiene, surgical technique, and standardized anesthetic protocols, among others. ${ }^{5}$ While this meta-analysis wasn't designed to assess most of these factors, we can gleam information from the surgical techniques and types of cases analyzed.

Of the 17 studies included in the meta-analysis, 4 assessed video-assisted thoracoscopic surgery lung resections only, and no differences were seen in postoperative complications in any study. Of the 8 studies that did demonstrate decreased postoperative complications, 3 included esophagectomy only, and 5 included mostly, if not entirely, open lung resections, 2 of which included pneumonectomies. This appears to suggest the greatest benefit of low tidal volume ventilation is to patients at greatest risk of postoperative complications 
(ie, esophagectomy, open lobectomy, and pneumonectomy). This may also suggest that centers that specialize in minimally invasive lung surgery may employ other modern perioperative, intraoperative, and postoperative measures as previously described, which combine to minimize risk of acute lung injury from OLV.

\section{References}

1. Lohser J, Slinger P. Lung injury after one-lung ventilation: a review of the pathophysiologic mechanisms affecting the ventilated and the collapsed lung. Anesth Analg. 2015;121:302-18.
2. Brassard CL, Lohser J, Donati F, Bussieres JS. Step-by-step clinical management of one-lung ventilation: continuing professional development. Can J Anaesth. 2014;61:1103-21.

3. The Acute Respiratory Distress Syndrome Network. Ventilation with lower tidal volumes as compared with traditional tidal volumes for acute lung injury and the acute respiratory distress syndrome. N Engl J Med. 2000;342:1301-8.

4. Peel J, Funk D, Slinger P, Srinathan S, Kidane B. Tidal volume during 1-lung ventilations: a systematic review and meta-analysis. J Thorac Cardiovasc Surg. 2022; 163:1573-85.e1.

5. Batchelor TJP, Rasburn NJ, Abdelnour-Berchtold E, Brunelli A, Cerfolio RJ, Gonzalez M, et al. Guidelines for enhanced recovery after lung surgery: recommendations of the Enhanced Recovery After Surgery (ERAS) Society and the European Society of Thoracic Surgeons (ESTS). Eur J Cardiothorac Surg. 2019;55: 91-115.
See Article page 1573 .

\section{Commentary: The tid(al)s are turning toward lower volumes}

\author{
Alexandra L. Potter and Chi-Fu Jeffrey Yang, MD
}

Previous literature has largely supported the use of lung protective ventilation (LPV) with lower tidal volumes in patients with acute respiratory distress syndrome (ARDS), finding that LPV with lower tidal volumes is associated with lower postoperative morbidity and mortality, likely through reduced stretch-induced lung injury and decreased release of inflammatory mediators. ${ }^{1,2}$

However, literature supporting the use of LPV with low tidal volumes in patients without ARDS during intraoperative 1-lung ventilation has lagged behind the ARDS literature. This may be a result of greater emphasis placed on sustaining normal $\mathrm{pH}$ and arterial carbon dioxide partial pressure rather than protecting the lung from injurious stretch in patients without ARDS. ${ }^{1,3}$ Recently, though, an increasing body of evidence shows that patients without

\footnotetext{
From the Division of Thoracic Surgery, Department of Surgery, Massachusetts General Hospital, Boston, Mass.

Disclosures: The authors reported no conflicts of interest.

The Journal policy requires editors and reviewers to disclose conflicts of interest and to decline handling or reviewing manuscripts for which they may have a conflict of interest. The editors and reviewers of this article have no conflicts of interest.

Received for publication Jan 10, 2021; revisions received Jan 10, 2021; accepted for publication Jan 11, 2021; available ahead of print Jan 19, 2021.

Address for reprints: Chi-Fu Jeffrey Yang, MD, Division of Thoracic Surgery, Department of Surgery, Massachusetts General Hospital, 55 Fruit St, Boston, MA 02114 (E-mail: cjyang@mgh.harvard.edu).

J Thorac Cardiovasc Surg 2022;163:1587-8

0022-5223/\$36.00

Copyright (c) 2021 by The American Association for Thoracic Surgery

http://dx.doi.org/10.1016/j.jtcvs.2021.01.040
}

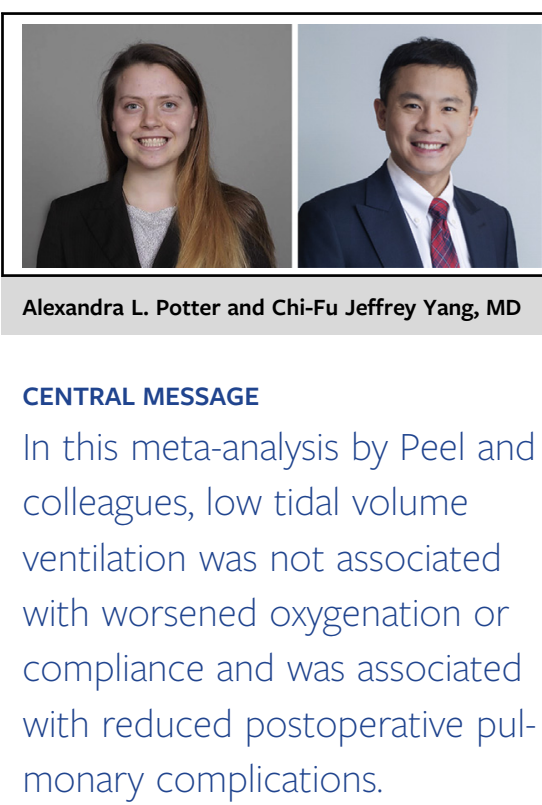

ARDS are susceptible to ventilator-induced lung injury and may benefit from LPV with lower tidal volumes. ${ }^{4-6}$

In this issue of the Journal, Peel and colleagues ${ }^{7}$ report a well-designed systematic review and random-effects metaanalysis of 18 studies to assess the effect of low tidal volume on perioperative outcomes. In total, 3963 patients who underwent pulmonary resection or esophagectomy with 1-lung ventilation were included. Of note, patients with severe preoperative pulmonary or cardiopulmonary disease were excluded. When compared with conventional tidal volumes $(8.1 \pm 3.1 \mathrm{~mL} / \mathrm{kg})$, low tidal volumes $(5.6 \pm 0.9 \mathrm{~mL} / \mathrm{kg})$ were associated with a decreased risk of developing postoperative pulmonary complications 